\title{
BioéthiqueOnline
}

\section{Na-no-body : de l'oubli du corps sensible en nanomédecine}

\section{Mathieu Noury}

Volume 5, 2016

URI : https://id.erudit.org/iderudit/1044278ar

DOI : https://doi.org/10.7202/1044278ar

Aller au sommaire du numéro

Éditeur(s)

BioéthiqueOnline

ISSN

1923-2799 (numérique)

Découvrir la revue

Citer cet article

Noury, M. (2016). Na-no-body: de l'oubli du corps sensible en nanomédecine. BioéthiqueOnline, 5. https://doi.org/10.7202/1044278ar

\section{Résumé de l'article}

Cet article expose les réflexions sur lesquelles se fonde un récent projet artistique intitulé Na-no-body, présenté à la galerie montréalaise Espace Projet dans le cadre de l'exposition Art + Bioéthique. Résultat d'une collaboration entre l'artiste Stephanie Coleman et le sociologue Mathieu Noury, ce projet souhaite contribuer au dialogue éthique sur le développement de la nanomédecine et de son rapport au corps. S'intéressant particulièrement à la notion de "médecine personnalisée " promue par la nanomédecine, cet article montre que loin de développer une approche réintégrant la personne et son expérience personnelle de la maladie au coeur du processus de soin, la nanomédecine apporte une réponse purement moléculaire et technique au soin. Plus spécifiquement, il est soutenu que deux idées principales fondent le modèle de prise en charge du patient promu par la nanomédecine : 1)

l'individualité du patient est pensée au regard du prisme de la pensée moléculaire ;2) la relation de soin, et donc au corps du patient, est hautement dépersonnalisée et technicisée, radicalisant la désindividualisation du soin propre à la biomédecine.
Ce document est protégé par la loi sur le droit d'auteur. L'utilisation des services d’Érudit (y compris la reproduction) est assujettie à sa politique d'utilisation que vous pouvez consulter en ligne.

https://apropos.erudit.org/fr/usagers/politique-dutilisation/ 


\title{
Na-no-body : De l'oubli du corps sensible en nanomédecine
}

\author{
TRAVAIL CRÉATIF / CREATIVE WORK \\ Mathieu Noury ${ }^{1}$
}

\author{
Reçu/Received: 9 Dec $2016 \quad$ Publié/Published: 16 Sept 2016 \\ Éditeurs/Editors: Jean-Christophe Bélisle-Pipon \& Lise Lévesque \\ Travail créatif/Creative work: Coleman S. Na-no-body. BioéthiqueOnline 2016;5/19
}

2016 M Noury, Creative Commons Attribution 4.0 International License

\begin{abstract}
Résumé
Cet article expose les réflexions sur lesquelles se fonde un récent projet artistique intitulé $\mathrm{Na-no-body,} \mathrm{présenté} \mathrm{à} \mathrm{la}$ galerie montréalaise Espace Projet dans le cadre de l'exposition Art + Bioéthique. Résultat d'une collaboration entre l'artiste Stephanie Coleman et le sociologue Mathieu Noury, ce projet souhaite contribuer au dialogue éthique sur le développement de la nanomédecine et de son rapport au corps. S'intéressant particulièrement à la notion de " médecine personnalisée " promue par la nanomédecine, cet article montre que loin de développer une approche réintégrant la personne et son expérience personnelle de la maladie au cœur du processus de soin, la nanomédecine apporte une réponse purement moléculaire et technique au soin. Plus spécifiquement, il est soutenu que deux idées principales fondent le modèle de prise en charge du patient promu par la nanomédecine : 1) l'individualité du patient est pensée au regard du prisme de la pensée moléculaire ; 2) la relation de soin, et donc au corps du patient, est hautement dépersonnalisée et technicisée, radicalisant la désindividualisation du soin propre à la biomédecine.
\end{abstract}

\section{Mots clés}

nanomédecine, nanotechnologies, médecine personnalisée, théranostique, corps, molécularisation, transhumanisme, art

\section{Summary}

This article presents the ethical reflexion of a recent art project, Na-no-body, presented at the Montreal gallery Espace Projet in the context of the exposition Art + Bioéthique. Na-no-body is the result of a collaborative work between the artist Stephanie Coleman and the sociologist Mathieu Noury. The aim of this project was to open an ethical dialogue on nanomedicine and its relationship with the body. To do so, the article examines specifically the notion of "personalized medicine" promoted by nanomedicine. This article proposes that, far from developing an approach reintegrating the person and the personal experience of illness at the heart of the care relationship, nanomedicine brings a simple molecular and technical response to caring. It is argued that two major themes constitute the core of this notion: 1) a molecular conception of personalization, a n d 2) a technical conception of personalization.

\section{Keywords}

nanomedicine, nanotechnoloy, personalized medicine, theranostics, body, molecularization, transhumanism, art

Affiliations des auteurs / Author Affiliations

${ }^{1}$ Université du Québec en Abitibi-Témiscamingue, Rouyn-Noranda, Canada

Correspondance / Correspondence

Mathieu Noury, mathieu.noury@uqat.ca

\section{Remerciements}

Je tiens à sincèrement remercier l'artiste Stephanie Coleman pour l'originalité et la créativité de son travail artistique. Je remercie également l'ensemble du comité de lecture de ce numéro spécial pour leur relecture attentive de l'article.

\section{Conflit d'intérêts}

Aucune declaré

\section{Acknowledgements}

I sincerely thank the artist Stephanie Coleman for the originality and creativity of her artwork. I also want to thank the editors of this special issue for their thorough re-reading of the article.

\section{Conflicts of Interest \\ None declared}

Et toutefois qui dit chair dit aussi sensibilité. Sensibilité, c'est-à-dire appropriation, mais appropriation intime, secrète, profonde, absolue de ma douleur à moi-même, et par conséquent, connaissance solitaire et unique de cette douleur.

Antonin Artaud, « Position de la chair » [1] 
À partir de la seconde moitié du $X X^{e}$ siècle, les avancées biomédicales vont profondément bouleverser les repères éthiques ordonnant nos rapports sociaux et individuels aux corps, à la vie et à la mort [2]. Pensons à quel point, dans les années 1950, la greffe d'organe et l'utilisation des respirateurs artificiels lors d'opérations cardiaques ont révolutionné la portée de l'intervention chirurgicale et la manière de penser la frontière entre la vie et la mort. Depuis lors, la révolution génétique et moléculaire en médecine, d'où émergent aujourd'hui les nanotechnologies et leurs promesses, a démultiplié la portée et les ambitions des innovations biomédicales, ainsi que les problèmes éthiques. Ceci contribuant à ouvrir une nouvelle ère culturelle dont l'un des piliers anthropologiques centraux est la croyance que désormais, grâce aux innovations technologiques et particulièrement aux innovations moléculaires, le biologique, le corps en lui-même n'est plus une limite à nos ambitions humaines. Croyance culturelle qui a enfanté de nouvelles idéologies comme le transhumanisme ${ }^{1}$. Considérant la nature humaine comme étant ontologiquement indéterminée, c'està-dire transgressant continuellement sa condition antérieure, le transhumanisme voit dans les innovations technologiques, et plus particulièrement dans les nanotechnologies, l'opportunité historique d'arracher l'Homme à des limites biologiques longtemps considérées immuables. Un exemple est le vieillissement, que le biogérontologue britannique et membre actif du mouvement transhumaniste, Aubrey De Grey, pense pouvoir retarder indéfiniment [3].

Fini le fatum comme sentiment intime de l'irréductible fatalité de la mort et de la fragilité de notre condition humaine. Nous expérimentons aujourd'hui une croyance collective en la puissance des innovations technologiques à repousser toutes les limites biologiques. La sagesse judéo-chrétienne de l'acceptation de la fragilité de la chair et de la finitude du corps s'est fait doubler par une éthique prométhéenne de l'infinie perfectibilité technique de la condition humaine [4]. Dieu n'est pas mort. II s'est déplacé vers les technosciences. Et son fils semble s'être incarné cette fois, non pas sous les traits d'un homme, mais sous ceux d'un préfixe, nano, dont la puissance performative suffit à générer instantanément, à chacune de ses annonces, des espoirs scientifiques et médicaux inouïs ainsi qu'à multiplier les millions de dollars en subvention de recherche. En effet, selon nombre de chercheurs en santé et d'experts en politique scientifique [5], le futur de l'art de guérir et de notre " mieux-être » se trouverait désormais du côté de ce nouveau domaine de la recherche biomédicale qu'est la nanomédecine et ses technologies moléculaires (nano-objets) capables de parcourir, d'analyser et de traiter les recoins les plus infinitésimaux du corps humain.

Généralement définie comme l'application des nanotechnologies aux soins de santé, la nanomédecine touche l'ensemble des champs d'intervention de la médecine, du diagnostic au traitement, grâce à la fabrication d'innovations moléculaires capables de cibler et de contrôler les processus biologiques à la base des maladies ${ }^{2}$. Les innovations nanomédicales incluent de nouveaux outils pour le diagnostic in vivo et in vitro (points quantiques, nanoparticules d'or, implants, laboratoire sur puce, etc.), de nouveaux dispositifs d'administration des médicaments (drug delivery systems), de nouveaux modes d'intervention combinant le diagnostic et la thérapeutique (théranostic) ou encore de nouvelles techniques d'ingénierie tissulaire. Ainsi la nanomédecine ne se comprend pas simplement comme un nouveau domaine biomédical qui viendrait se greffer à ceux déjà existants. Elle représente plutôt un nouveau paradigme des soins de santé qui promeut une nouvelle approche de la prise en charge du patient et de l'intervention médicale intégralement dépendante des technologies moléculaires [6].

\footnotetext{
${ }^{1}$ L'ensemble des détails concernant les thèses soutenues par le mouvement transhumaniste sont disponibles sur le site Internet de la World Tanshumanist Association.

2 L'Office québécois de la langue française a proposé la définition officielle suivante de la nanomédecine: « domaine consacrée à la santé qui utilise les connaissances acquises en médecine, en biologie et nanotechnologie pour le plus souvent fabriquer, à l'échelle des molécules et des cellules, des outils aux dimensions nanométriques servant habituellement à diagnostiquer ou à traiter des maladies, à administrer des médicaments ou à réparer, à reconstruire ou à remplacer des tissus ou des organes. "
} 
C'est précisément cette nouvelle approche biomédicale que l'artiste Stéphanie Coleman et moi-même avons décidé d'explorer dans le cadre de notre collaboration pour l'exposition Art + Bioéthique à la galerie montréalaise Espace Projet. Nous avons décidé de travailler sur ce vaste et complexe sujet sous l'angle particulier de la manière dont est abordé le corps en nanomédecine. Trop souvent, la nanomédecine et, plus largement, les nouvelles techniques issues des biotechnologies sont interrogées sous leur versant le plus médiatique, futuriste et radical, soit les possibilités de transformation et d'augmentation technologique du corps humain, chères aux transhumanistes. Nombre d'analystes [7-11] et d'artistes (ex. : Eduardo Kac, Stelarc, Natasha Vita More, Thierry Mutin) travaillent sur cette dernière question. II semblait donc opportun de dévier de cette trajectoire saturée, et largement déconnectée du développement pratique de la nanomédecine dans les laboratoires de recherche, pour aborder certains problèmes liés au rapport concret à l'intimité du corps qui est caractéristique du modèle de soin biomédical promu par la nanomédecine. Ce corps qui, nous l'aborderons plus loin, est objectivé en véritable champ de bataille où s'affairent les " missiles moléculaires » de la nanomédecine, accordant peu de considération au corps vécu et sensible du malade. D'où le choix du titre Na-no-body pour notre projet. Celui-ci souligne ironiquement ce rapport d'objectivé, cet oubli du corps vécu porté par le regard moléculaire et guerrier de la nanomédecine, dont l'effet est d'évacuer toute référence au corps sensible de la personne malade qui est pourtant le lieu même de l'expérience intime de sa maladie. En ce sens, au-delà de ses nouveautés technologiques, la nanomédecine s'inscrit dans la continuité de la médecine scientifique occidentale travaillant sur un corps médical désubjectivisé, désindividualisé. Un corps vide d'être et de sensibilité.

Le présent texte vise à exposer les réflexions sur lesquelles se fonde notre projet artistique Na-nobody afin de contribuer au dialogue éthique sur le rapport au corps en nanomédecine et, plus largement, dans le contexte de ce que John Pickstone appelle la " technomédecine » [12] pour souligner l'univers contemporain des soins de santé marqué par la dominance d'un modèle d'ingénierie biomédicale axé sur le contrôle et la transformation technique du corps et de la vie en elle-même, plus que sur un rapport humain d' "attention " - une éthique du care [13] - envers la personne malade. Ce modèle d'ingénierie biomédicale se développe sur la profonde transformation du «style de pensée » de la biomédecine que le sociologue Nikolas Rose a conceptualisé avec son concept de "molécularisation » [14]. Se trouvant au fondement du développement de la nanomédecine, ce large mouvement de fond épistémologique repose sur la concentration simultanée du regard scientifique vers l'échelle des mécanismes moléculaires et de la finalité de la pratique médicale vers la transformation technique de ces mécanismes, mettant de côté l'« attention » thérapeutique envers le patient en faveur du succès technique de l'intervention médicale [15].

\section{Le projet, sa démarche, son " éthique »}

Na-no-body repose sur la création, d'une part, d'une tapisserie " sensible ", foisonnante d'éléments décoratifs rappelant une flore étrange et attirante qui représente la complexité et la sensibilité de l'intimité organique du corps vécu, et, d'autre part, des boîtes de Pétri. À l'intérieur de ces dernières se trouve de petites formes curieuses, inquiétantes, voire menaçantes renvoyant à une représentation imaginée, visible à l'œil humain, à la fois du corps objectivé/molécularisé de la nanomédecine et de ses nano-objets parcourant le corps, tel des " missiles magiques » (magic bullets), pour reprendre la formule prophétique employée au tout début du $\mathrm{XX} \mathrm{X}^{\mathrm{e}}$ siècle par le médecin allemand Paul Ehrlich [16]. Formule aujourd'hui largement reprise par les promoteurs de la nanomédecine. Les petites formes étranges jouent volontairement de l'ambigüité entre corps objectivé/molécularisé et nano-objets. Elles représentent symboliquement un concentré ambigu et inquiétant d'une compréhension purement technoscientifique, objectivée/molécularisée, distante et désindividualisée du corps et de la forme de l'intervention bio(nano)médicale.

Le public entre dans une pièce exiguë, à la lumière tamisée, presque intégralement recouverte par la tapisserie et ses motifs floraux foisonnants. Les boîtes de Pétri contenant les objets ambigus parsèment la pièce, créant un effet de rencontre entre la représentation sensible du corps qu'évoque 
la tapisserie et celle du corps étranger et distant de la (nano)médecine symbolisée par les boîtes et leurs contenus. Cette rencontre se veut une réflexion sur la distance entre la réalité vécue du corps sensible de la personne malade et la compréhension objective, moléculaire, technoscientifique du corps tel qu'il est expérimenté par le regard et la pratique (nano)médicale. Je mets ici « nano » entre parenthèses, car cette logique de mise à distance volontaire du corps sensible n'est pas propre à la nanomédecine, mais plus largement au regard biomédical lui-même qui fonde cette expérience technoscientifique du corps.

Une fois dans la pièce, le public est invité à toucher la tapisserie " sensible » dont certains espaces sont dotés de petits capteurs déclenchant des lumières. Ce dispositif simple souhaite favoriser une expérience tactile avec la tapisserie, une expérience de proximité et d'intimité avec celle-ci où le public entre en relation avec la flore foisonnante, représentant la complexité et la sensibilité de notre " végétation » intérieure.

Cette démarche artistique permet, modestement, de questionner les usages des nouvelles pratiques médicales d'intervention technologiques dans le corps sans s'immiscer dans celui-ci et sans le transformer. Ce point a son importance éthique, car plusieurs des projets et réflexions artistiques qui se sont emparés de la question des nouvelles technologies biomédicales, spécifiquement en bioart et bodyart, ont donné lieu à des formes d'interventions intrusives et transformatives sur le corps et le vivant. Notre point de vue présuppose une responsabilité éthique [17] fondée sur un respect de la fragilité et de la sensibilité du corps et du vivant. L'avant-gardisme artistique et le progressisme de la pensée ne nécessitent nullement d'intervenir sur le corps et le vivant pour réfléchir sur la spécificité, les effets et la portée des innovations technologiques sur ceux-ci. Pas besoin de se faire Prométhée et de s'introduire en colonisateur amateur dans les territoires de la vie organique et moléculaire pour réfléchir sur les profondes mutations socioculturelles et identitaires liées aux potentialités des nouvelles technologies biomédicales.

\section{Le projet et ses réflexions}

Na-no-body se fonde sur les analyses issues de mes travaux sur la nanomédecine $[18,19]$ et sur de nombreuses entrevues que j'ai réalisées auprès de chercheurs dans ce domaine [20]. Ce projet artistique reprend plus particulièrement mes réflexions sur la manière dont la nanomédecine pense et développe l'idée d'un soin dit «personnalisé ». En effet, l'une des grandes promesses de la nanomédecine est d'être une " médecine personnalisée ». Elle promet des outils de diagnostic et de thérapeutique entièrement personnalisés pour les besoins de chaque patient et incarne le développement d'une approche médicale se voulant patient-friendly, c'est-à-dire plus proche des besoins des personnes malades.

Loin de développer une approche réintégrant la personne et son expérience personnelle de la maladie au cœur du processus de soin, la nanomédecine apporte une réponse purement moléculaire et technique au soin. Je vais m'attarder brièvement sur deux idées principales qui fondent ce constat : 1) l'individualité du patient est pensée au regard du prisme de la pensée moléculaire et 2) la relation de soin, et donc au corps du patient, est hautement dépersonnalisée et technicisée, radicalisant la désindividualisation du soin propre à la biomédecine.

\section{Le patient comme individualité " moléculaire »}

La conception du soin en nanomédecine rejette d'emblée toute dimension interpersonnelle ou psychologique. Le soin se comprend au regard du prisme des particularités moléculaires de la personne malade. L'idée est que grâce à l'identification moléculaire chaque patient pourrait avoir une thérapeutique façonnée sur mesure, selon son "individualité ». Les dispositifs de diagnostic moléculaire offrent en effet la possibilité d'avoir un certain nombre d'informations, les marqueurs moléculaires ou biomarqueurs, dont le point de référence centrale est l'individu lui-même. La 
normalité (santé) et l'anormalité (pathologie) sont considérées comme pouvant être mesurées à partir des régularités et des variations moléculaires propres à un individu donné [21].

Le soin se comprend donc en rapport à une conception de l'individualité du patient définie sur la base des caractéristiques moléculaires de la personne malade. En ce sens, l'individualité moléculaire est le point de départ à partir duquel est pensé le soin. C'est sur la base de ce réductionnisme moléculaire que s'élaborent les nouvelles possibilités thérapeutiques dites " personnalisées » en nanomédecine. Nous nous trouvons ici dans la continuité directe de la biomédecine qui s'est historiquement développée au travers de la rationalisation du corps du patient et de la mise de côté de son expérience individuelle de la maladie. Ce que le chirurgien et historien de la médecine Charles Masquelet appelle "l'oubli du sujet» [22]. Cet oubli du sujet prend aujourd'hui la forme d'une nouvelle figure rationalisée du malade qui est principalement pensée au travers de données moléculaires, ce que nous avons tenté de symboliser avec le dispositif artistique de Na-no-body.

Loin de signifier la réintroduction de ce que Georges Canguilhem considérait être la réalité individuelle et qualitative de la maladie [23], cette conception de l'individualité moléculaire s'effectue sur la base d'un renouvellement du modèle biomédical et du rejet de l'expérience du patient, alors même que la prise en compte de cette expérience constitue un élément essentiel de la guérison et de la relation patient-médecin. Le patient et son expérience sont dilués dans des formes d'identité nosologiques issues de catégories moléculaires. La figure du patient renvoie à un individu réifié à partir de ses caractéristiques moléculaires. Une telle conception du soin repose sur un important paradoxe: "C'est en se faisant la plus scientifique et objective possible que cette médecine veut devenir adaptée à la situation unique de chaque patient » [24].

\section{Une prise en charge du patient purement technique}

Fondée sur le présupposé selon lequel l'accroissement du degré de contrôle technique des composantes biologiques élémentaires est le moyen privilégié pour améliorer la pratique biomédicale, la nanomédecine a pour objectif central l'amélioration des capacités de contrôle technique de ses dispositifs nanomédicaux. Le soin se comprend non seulement au regard de l'individualité moléculaire, mais aussi, en même temps, au regard de l'accroissement du degré de complexité et de maîtrise technique des technologies nanomédicales. Par là même, le soin ne signifie nullement de trouver une meilleure balance entre la nécessité de rationalisation technique de l'acte médical et la prise en compte de l'expérience individuelle de la maladie.

Le nouveau de concept de "théranostic » (thérapeutique + diagnostic) illustre parfaitement cette conception à la fois moléculaire et « hypertechnicisée » du soin. Celui-ci vise à la création de vecteurs nanoparticulaires ou nanoplateformes multifonctions capables, lors d'une même procédure, de réaliser un test diagnostique, de délivrer un traitement à une cible précise et de suivre la réponse à ce même traitement. Les propriétés physicochimiques de la nanoparticule permettent à celle-ci de traverser certaines parties du corps, comme l'endothélium vasculaire, en se rendant indétectable par les macrophages du foie, de la rate et de la moelle osseuse, et de reconnaître un tissu pour s'adresser à lui, comme une clé dans une serrure. Ces "vecteurs furtifs " [25] se déplacent dans le corps en déjouant les mécanismes de défense biologiques.

En nanomédecine, les métaphores guerrières abondent (missiles thérapeutiques, nano-weapons, bombes intelligentes, etc.) et rendent compte de la traduction du problème thérapeutique en termes strictement d'ingénierie [26]. Impossible ici de ne pas faire le parallèle avec la figure du drone, cet instrument d'une "violence à distance » [27] qui, loin de se cantonner aux champs de bataille de la guerre moderne, semble se retrouver aujourd'hui au cœur des nouvelles stratégies en santé. Le " théranostic " renvoie ainsi à une conception du soin proprement technoscientifique qui se développe au travers de la diffusion de métaphores guerrières servant à mettre en valeur la capacité de contrôle, de surveillance et d'efficacité technique des dispositifs nanomédicaux. 
Devenu le théâtre d'une mission guerrière se déroulant à distance, le corps du patient se pense par la médiation d'un nanodispositif, à qui est délégué la capacité d'agir, laissant place à une relation au patient et donc au corps, entièrement définie technologiquement et déshumanisée. Encore une fois, il ne s'agit pas d'une rupture avec la tradition biomédicale moderne. La biomédecine s'est développée au travers d'une mise à distance graduelle du chirurgien ou du médecin et de leur perception subjective, comme l'acte du toucher qui devient aujourd'hui de plus en plus rare. La modernité médicale a signifié la maîtrise et l'inhibition progressive du geste chirurgical et médical par sa mécanisation ainsi que sa standardisation, dont l'instrument médical va s'imposer comme le moyen de contrôle privilégié [28]. La mise à distance progressive du médecin et de son ressenti subjectif par la rationalisation de la pratique médicale a constitué historiquement la garantie de sa scientificité et marqué l'émergence du rôle central de l'instrument comme médium de contrôle de la pratique.

La postmodernité médicale se comprend alors comme la délégation systématique de l'agir humain face aux dispositifs technologiques. La représentation du progrès médical est désormais associée au développement d'une médecine entièrement technicisée où le retrait du geste et du corps à corps apparaît comme la garantie de l'amélioration du soin. Les nanoplateformes de " théranostic » sont caractéristiques de cette mise à distance technologique et de cette croyance en la supériorité d'une action technologique écartant la subjectivité de l'agir humain. Tout rapport humain et sensible au corps est effacé.

\section{Conclusion}

Na-no-body repose sur le constat critique suivant : le modèle de prise en charge du patient promu par la nanomédecine conduit à un décentrement radical de la personne malade et de son expérience de la maladie. Un tel modèle se trouve en opposition avec nombre d'analyses en sciences humaines et sociales qui ont démontré l'importance de la prise en compte de l'expérience vécue de la maladie [29]. Ces analyses ont montré que la maladie n'est pas uniquement vécue comme une pathologie physiologique, mais aussi une expérience existentielle sur le sens même de ce qui arrive à la personne malade et provoque de profondes questions sur le sens de sa vie. Bien que la biomédecine ait construit sa légitimité sur son indéniable efficacité technique, elle a tendance à oublier que la maladie combine des dimensions à la fois physiologiques et existentielles qui sont liées à l'expérience de la souffrance [30]. Elle oublie par là même que la maladie est d'abord perçue par une subjectivité, s'incarnant dans un corps éminemment sensible, avant d'être conçue par le diagnostic et prise en charge par le système médical [31]. Et peut-être, encore plus profondément, elle ne prend en considération qu'une seule facette du sens du mot " soin » : celle du soin comme techné ou therapeia, c'est-à-dire comme l'application pratique des concepts et techniques de la médecine. L'autre facette du soin reste relativement absente des développements biomédicaux : celle du soin comme epimeleia [22], comme relation d'accompagnement, relation d'aide envers l'autre, permettant de donner un sens proprement humain à la relation médicale et donc à l'expérience que fait le malade de son passage dans le monde médical. Ainsi, au regard de la place de plus en plus importante prise par cette nouvelle médecine techno-moléculaire, il devient encore plus urgent pour la biomédecine de trouver un meilleur équilibre entre ses ambitions de contrôle technique du corps et l'attention à la réalité subjective et sensible de l'expérience de la maladie.

\section{Références}

1. Artaud, A. (1956), L'ombilic des Limbes, Paris, Gallimard.

2. Lafontaine, C. (2008), La société post-mortelle. La mort, l'individu et le lien social à l'ère des technosciences, Paris, Seuil.

3. Lain, D. (2016), Advancing Conversations: Aubrey De Grey - Advocate For An Indefinite Human Lifespan, UK, Zero Books.

4. Le Dévédec, N. (2015), La société de l'amélioration. La perfectibilité humaine des Lumières aux transhumanisme, Montréal, Liber. 
5. Instituts de Recherche en Santé du Canada : Médecine régénératrice et nanomédecine. Investir aujourd'hui dans la promesse de demain (2006, 2009).

6. Noury, M. \& Lafontaine, C. (2014), De la nanomédecine à la nanosanté : vers un nouveau paradigme biomédical, Socio-Anthropologie, 29, 13-36.

7. Michaud, Y. (2002), Humain, inhumain, trop humain, Paris, Climats.

8. Lecourt, D. (2003), Humain, posthumain. La technique et la vie, Paris, PUF.

9. Robitaille, A. (2007), Le nouvel Homme nouveau. Voyage dans les utopies de la posthumanité, Québec, Boréal.

10. Hoquet, T. (2011), Cyborg philosophie. Penser contre les dualismes, Seuil, Paris.

11. Besnier, J.-M. (2012), Demain les posthumains. Le futur a-t-il encore besoin de nous ?, Paris, Pluriel.

12. Pickstone, J. (2001) Ways of Knowing: A New History of Science, Technology, and Medicine, Chicago, Chicago University Press.

13. Gilligan, C., (2008), Une voix différente, Paris, Flammarion.

14. Rose, N. (2007), The Politics of Life Itself: Biomedicine, Power, and Subjectivity in the TwentyFirst Century, Princeton, Princeton University Press.

15. Kay, L. E. (2000), Who Wrote the Book of Life? A History of the Genetic Code, Stanford, Stanford University Press.

16. Strebhardt, K. \& Ullrich, A. (2008), Paul Ehrlich's Magic Bullet Concept: 100 Years of Progress, Nature Reviews Cancer, 8, 473-480.

17. Jonas, H. (1990), Le principe responsabilité. Une éthique pour la civilisation technologique, Paris, Champs.

18. Noury, M. \& Lopez, J. (2016), Nanomedicine's Transversality: Some Implications of the Nanomedical Paradigm, Wireless Computing in Medicine: From Nano to Cloud with Ethical and Legal Implications, Eshaghian-Wilner, M. (ed.), New Jersey, Wiley.

19. Noury, M. (prévu pour 2016), La nanosanté. Perspective et enjeux sociologiques de la révolution des nanotechnologies en médecine, Montréal, Liber.

20. Noury, M. (2014), La nanosanté. Perspective et enjeux sociologiques de l'application des nanotechnologies à la médecine, Thèse de doctorat, Montréal/Paris, Université de Montréal/ Université Paris Ouest-Nanterre.

21. Boenink, M. (2010), Molecular Medicine and Concepts of Disease: The Ethical Value of a Conceptual Analysis of Emerging Biomedical Technologies, Medicine, Health Care and Philosophy, 13 (1), 11-23.

22. Masquelet, A.-L. (2010), Médecine contemporaine et disposition au soin, La philosophie du soin. Éthique, médecine et société, Benaroyo, L. \& al. (dir.), Paris, PUF, 205-217

23. Canguilhem, G. (2009), Le normal et le pathologique, Paris, PUF.

24. Dion-Labrie, M.; Fortin, M.C.; Hébert, M.J.; Doucet, H. (2008), Réflexions éthiques sur la médecine personnalisée: I'alliance de la science et de la médecine enfin réalisée?, Revista Colombiana de Bioética, 3 (2), 33-56.

25. Couvreur, P. (2010), Les nanotechnologies peuvent-elles contribuer à traiter des maladies sévères?, Leçon inaugurale de la Chaire d'innovation Technologique Liliane Bettencourt 2009-2010, Collège de France.

26. Bensaude-Vincent B. \& Loeve S. (2013), Metaphors in Nanomedicine: The Case of Targeted Drug Delivery, Nanoethics, 8(1), 1-17.

27. Chamayou, G. (2013), Théorie du drone, Paris, La fabrique.

28. Reiser, S. J. (2009), Technological Medicine: The Changing World of Doctors and Patients, Cambridge, Cambridge University Press.

29. Pierret, J. (2003), The IIIness Experience: State of Knowledge and Perspectives for Research, Sociology of Health and IIIness, 24, 4-22.

30. Good, B. (1994), Medicine, Rationality and Experience, Cambridge, Cambridge University Press.

31. Le Blanc, G. (2010), L'expérience de la vie malade, La philosophie du soin. Éthique, médecine et société, Benaroyo L. \& al. (dir.), Paris, PUF, 301-317. 